\title{
Longitudinal follow-up Study of the Intrarenal Hemodynamics and Pulmonary Function in Patients with T2DM
}

\author{
Lian Qun Jia ${ }^{1 *}$, Xiao Lin Jiang ${ }^{1}$, Wei Chen ${ }^{1}$, He Tai ${ }^{1,2}$ and Jin Song Kuang ${ }^{3}$ \\ ${ }^{1}$ Key Laboratory of Ministry of Education for Traditional Chinese Medicine Visera-State Theory and Application, Liaoning University of Traditional Chinese \\ Medicine, China
}

${ }^{2}$ Department of Endocrinology and Metabolic, Liaoning Provincial Corps Hospital of Chinese People's Armed Police Forces, Shenyang, China

${ }^{3}$ Department of Endocrinology and Metabolic, Shenyang the Fourth Hospital of People, Shenyang, China

*Corresponding author: Lian Qun Jia, Liaoning University of Traditional Chinese Medicine, Huanggu District, Chongshan Road No. 79, Shenyang, Liaoning, 110847, China.

Received Date: February 25, 2020

Published Date: March 10, 2020

\begin{abstract}
Purpose: The main aim of this study is to long-range assess incidence of diabetic kidney disease (DKD) and pulmonary function in patients with type 2 diabetes mellitus (T2DM) during 2000 and 2015.

Methods: 60 subjects newly diagnosed with T2DM were divided into two groups according to their arterial resistivity index (RIs) ( $\leq 0.7$ and $>0.7$ ). These patients underwent tests designed to assess the early changes in pulmonary function and intrarenal haemodynamics associated with diabetes, the results of which were compared to corresponding test results for the same group of patients between 2000 and 2015. The endpoints of the study were the between-group differences in the changes in the pulmonary functional parameters, the mean kidney arterial RI in the bilateral interlobular renal arteries, and the indicated renal functional parameters (BUN, Cr, AER, and UACR).

Results: The subjects with RIs $\leq 0.7$ displayed significantly better pulmonary function in the year 2000 than subjects with RIs $>0.7$. Moreover, subjects in the former group displayed improvement and smaller decreases in their pulmonary functional parameters from 2000 to 2015 compared to subjects in the latter group. Additionally, subjects in the former group displayed improvement and smaller increases in their renal functional parameters, i.e., their BUN and Cr, and their AERs and UACRs from 2000 to 2015 compared to subjects in the latter group. The incidence of DKD in the group with RIs $\leq 0.7(6 / 25,24.00 \%)$ was significantly lower than that in the group with RIs $>0.7(23 / 45,65.71 \%)(\mathrm{P}<0.05)$.

Conclusion: The renal functional parameters, the right kidney RI may also serve as a predictor of diabetes-related changes in pulmonary function and renal injury in the future.

Keywords: Type 2 diabetes mellitus; Diabetic kidney disease; Pulmonary function; Intrarenal haemodynamics; Resistivity index

Abbrevations: T2DM: Type 2 Diabetes Mellitus; DN: Diabetes Nephropathy; DKD: Diabetes Kidney Disease; ESRD: End-Stage Renal Disease; UACR: Urinary Albumin/Creatinine Ratio; AER: Albumin Excrete Rate; HbA1c: Glycosylated HemoglobinA1c; FBG: Fasting Plasma Glucose; 2hPBG: 2-Hour Postprandial Blood Glucose; RBS: Random Blood Sugar; OGTT: Oral Glucose Tolerance Test; VC: Vital Capacity; FVC: Forced Vital Capacity; FEV1: Forced Expiratory Volume in 1 Second; PEF: Peak Expiratory Force; MVV: Maximal Voluntary Ventilation; TLC: Total Lung Capacity; FEV1/ FVC: Forced Expiratory Volume in 1 Second/ Forced Vital Capacity; DLCO: Diffusing Capacity for Carbon Monoxide of Lung; DLCO/VA: Diffusing Capacity for Carbon Monoxide of Lung/Unit Volume; CDU: Color Doppler Ultrasound; DR: Diabetes Retinopathy; PSV: Peak Systolic Velocity; EDV: End Diastolic Velocity; RI: Resistivity Index; SD: Standard Deviation; BUN: Blood Urea Nitrogen; Cr: Creatinine; TC: Total Cholesterol; HDL-C: High Density Lipoprotein Cholesterol; LDL-C: Low-density lipoprotein cholesterol; TG: Triglycerides; SBP: Systolic blood pressure; DBP: Diastolic blood pressure; BP: Blood pressure; LS: least squares; CMECs: cardiac microvascular endothelial cells; OAPP: advanced oxidation protein products; GFR: glomerular filtration rate; K/DOQI: Kidney Disease Outcome Quality Initiatives; AGEs: Advanced Glycated End Products; RAS: Renin Angiotensin System
\end{abstract}

\section{Introduction}

The prevalence of T2DM is increasing worldwide, particularly in Asian countries [1]. Patients with T2DM develop abnormalities

related to glucose and lipid metabolism, phenomena associated with multiorgan dysfunction. Moreover, T2DM has been identified 
as an independent risk factor for cardiovascular disease, as affected patients have a two-fold higher risk of developing cardiovascular disease than unaffected patients [2], and leads to the development of vascular diseases such as DN and DR [3], which are the leading causes of end-stage renal disease (ESRD) and acquired blindness, respectively [4]. New terminology describing kidney disease attributable to diabetes has been introduced in recent guidelines (National Kidney Foundation, 2007), which stipulate that the term 'DN' should be replaced by 'DKD', a major long-term microvascular complication of diabetes characterized by functional, structural and clinical abnormalities of the kidneys caused by diabetes that is highly prevalent among patients with the disease.

More than 3 decades ago, researchers established that patients with T2DM had less alveolar gas exchange capacity than healthy subjects [5]. However, hyperglycaemia-induced pulmonary vascular injury is a complication of T2DM that has been overlooked by researchers attempting to devise treatments for the numerous complications associated with the disease. Obesity, smoking, vascular disease, and prolonged diabetes are also significant contributors to reductions in lung function, and ex-smokers display clinically significant chronic air flow obstruction [6].

Colour doppler ultrasound (CDU), a modality that is widely used in a variety of medical fields, evaluates blood flow velocity based on shifts in Doppler signals [7]. Patients with hyperglycaemia and uncontrolled blood pressure have significantly increased blood flow compared to patients with systemic hypertension without diabetes (as estimated by CDU) [8]. The Doppler RI [PSV - PED/ PSV] that reflects intrarenal vascular resistance has been widely used to quantify the alterations in renal blood flow that may develop in renal disease [9].

To the best of our knowledge, no studies have used the intrarenal haemodynamics (RI) to predict changes in pulmonary function and intrarenal haemodynamics in patients with T2DM without DKD.

\section{Methods}

\section{Subjects}

The patients were included in the study: 1) patients with a diagnosis of T2DM, as defined by the guidelines of the American Diabetes Association [10]; 2) patients with no history of smoking, pulmonary disease, or recent viral illnesses; 3) patients without hepatopathy, nephropathy, hyperuricaemia, or gastrointestinal disease; and 4) patients likely to comply with the guidelines of the study who were able to visit our hospital for periodic assessments. The patients were excluded from the study: 1) patients with T1DM, as well as patients with gestational diabetes and patients who were lactating; 2) patients with inadequate renal function and other renal conditions that may affect intrarenal haemodynamics, such as urolithiasis, urinary infections, and large renal cysts; 3) patients whose liver function were abnormal or with the class III or IV heart failure or a history of coronary angioplasty, or myocardial infarction within 6 months before enrolling in the study; 5) patients with DKD and hypertension (patients receiving antihypertensive drugs).

\section{Study design}

154 adult patients with T2DM (82 males and 72 females) were initially diagnosed with the disease in 2000 and were subsequently divided into two separate groups according to their RIs $(\leq 0.7$ or $>0.7$ ); however, we were able to locate only 60 of these patients (32 males, 28 females) in 2015. These patients' pulmonary functional parameters, RIs, UACRs, AERs, and BUN and Cr levels were measured in 2015 and compared to corresponding values measured in 2000.

\section{Study assessments and endpoints}

Blood specimen collection and laboratory testing: Venous blood samples were collected between 6 and 8 AM following a fasting period of at least 8 hours and used for measurements of FPG levels, HbA1c levels, and renal functional parameters (BUN, Cr). For this procedure, $5 \mathrm{ml}$ of venous blood was placed in a glass tube, stored at room temperature for at least $10 \mathrm{~min}$, and then centrifuged (3000 r/min) for $10 \mathrm{~min}$ to separate the plasma from the serum, the latter of which was stored at $-70{ }^{\circ} \mathrm{C}$ in a cryogenic refrigerator. BUN and $\mathrm{Cr}$ levels were measured according to the instructions of the corresponding research kits. All the specimens were used for measurements of the above parameters within 1 week of collection.

Urine sample collection and laboratory tests: Urine output was quantified via a single 24 -h urine collection. Urinary albumin concentrations were measured using a double-antibody radio immunoassay with a sensitivity of $0.5 \mathrm{mg} / \mathrm{l}$, an intra-assay coefficient of variation of $4.5 \%$, and an inter assay coefficient of variation of $5.3 \%$ within the range of $10-50 \mathrm{mg} / \mathrm{l}$.

Pulmonary function measurements: The indicated pulmonary functional parameters (VC\%, FVC\%, FEV1\%, PEF\%, MVV\%, TLC\%, the FEV1/FVC\% ratio, DLCO\%, and the DLCO/VA\% ratio) were measured using a spirometer. We used the measuredto-expected value ratios and the percentages of the predicted value to eliminate the influence of age, height, and weight on the results. The subjects were asked to remain seated and rest quietly for at least $30 \mathrm{~min}$ before testing. The pulmonary function tests were performed 3 times, and the best of 3 acceptable readings for each parameter was used in the analysis. Spirometry and pulmonary functional analysis were performed by trained professionals.

Intrarenal haemodynamic parameter measurements: We measured the indicated intrarenal haemodynamics parameters (PSV, EDV, and RI) in the bilateral interlobular renal arteries of subjects who had fasted for at least $8 \mathrm{~h}$ by Doppler sonography after documenting the subjects' blood pressures and pulse rates. The examinations were performed after the subjects had rested for $15 \mathrm{~min}$ and with each subject in the supine position, as described previously. Colour duplex Doppler sonography was used to examine the vasculature of the left and right renal parenchyma and the 
main trunk of the renal artery. If no abnormalities in kidney size or vasculature structure were noted, 3 pulsed Doppler measurements were initiated in the interlobar arteries located at the center of the kidney, as well as in the arteries located at its upper and lower poles, within 5 minutes. The pulsed Doppler sampling gate was located in the interlobar arteries, and the angle of insonation, which was measured as precisely as possible, was kept under $60^{\circ} \mathrm{C}$. The PSV and EDV were documented in centimeters per second, and the RI was calculated as (PSV - EDV) / PSV. For each recording, the RI was measured only when at least 3 consecutive waveforms with similar appearances were observed. A mean of 3 RI measurements was obtained for each kidney. All examinations were performed in duplicate by the same operator, who had no knowledge of the two groups (diabetes or control) $[11,12]$.

\section{Statistical analysis}

Measurement data were expressed as the mean \pm SD, and numerical data were expressed as percentages. Statistical analysis was conducted using the SPSS statistical package (Version 17.0, SPSS Inc. Chicago, IL, USA). Differences in categorical variables between the two groups were evaluated using the chi-square test; differences in continuous variables between the two groups were evaluated using the independent-samples t test; before and after treatment within-group differences in continuous variables were assessed using the paired-sample $t$ test. $\mathrm{P}<0.05$ was considered statistically significant.

\section{Results}

Pulmonary and renal function were assessed in patients with T2DM who were organized into separate groups according to their RIs. Subjects with RIs $\leq 0.7$ had significantly better pulmonary (VC\%, FVC\%, FEV1\%, PEF\%, MVV\%, TLC\%, the FEV1/FVC\% ratio, DLCO \%, and the DLCO/VA\% ratio), renal function (the AER and UACR and Cr, and BUN) in 2000 than subjects with RIs >0.7. Moreover, subjects in the former group displayed improvements and smaller decreases and increases in their pulmonary and renal functional parameters between 2000 and 2015, respectively, compared to subjects in the latter group. Additionally, the incidence of DKD in the group with RIs $\leq 0.7(6 / 25,24.00 \%)$ was significantly lower than that in the group with RIs $>0.7(23 / 35,65.71 \%)(\mathrm{P}<0.05)$ (Table 1$)$.

\section{Discussion}

The results of this study indicate that in addition to renal functional parameters, the combination of the right kidney RI and the GFR may also be a good predictor of changes in pulmonary function in patients with diabetes, as well as a more sensitive indicator of changes in pulmonary function in such patients during the pre-clinical stages of DKD than the UACR or AER.

Renal Doppler RIs are widely used to evaluate blood flow in renal parenchymal diseases. RIs, which reflect intrarenal vascular resistance, a measure of vascular compliance [9], are also widely used to quantify changes in renal blood flow that may be attributable to renal diseases. Interestingly, previous studies have shown that renal Doppler sonography is an effective noninvasive and inexpensive means of screening for renovascular hypertension correctable via treatment with captopril [13]; therefore, we evaluated intrarenal haemodynamics by examining the RI using Doppler sonography.

The mechanisms underlying the occurrence of lung damage in diabetes are not fully understood. Thus, hyperglycaemia damages the lung, collagen is less susceptible to proteolysis because of nonenzymatic glycosylation of proteins in the lungs and chest wall, leading to its accumulation in lung connective tissue. This process is triggered mainly by hyperglycaemia and is thus more pronounced in patients with poor glycaemic control than in patients with good glycaemic control. In addition, nonenzymatic glycosylation of proteins in the lungs decreases lung compliance $[14,15]$. Clinically, loss of microvascular reserve in the lung may be associated with an increased risk of hypoxia in acute or chronic pathological lung conditions, such as pneumonia, asthma, chronic obstructive pulmonary disease, and congestive heart failure. Moreover, microvascular abnormalities frequently contribute to histological changes in the lung parenchyma, such as nodular fibrosis [15].

Systemic inflammation is another concern in patients with diabetes. Systemic inflammation induced by oxidative stress is associated with endothelial dysfunction in patients with diabetes [16-18]. Additionally, insulin resistance can alter lung volume and mechanical function via mediators such as leptin [15] and may independently cause airflow obstruction in a manner similar to that in which peripheral airway inflammation causes air flow obstruction in asthma [19]. Lung CO transfer capacity is significantly affected by the integrity of the lung capillary endothelium, a finding that supports that idea that clinicians should devote more attention to pulmonary vascular changes. The reports on lung function testing in patients with diabetes that have been published during the last 15 years have focused predominantly on pulmonary microangiopathy. The lung functional parameters that are related specifically to pulmonary microangiopathy include pulmonary capillary blood volume and CO transfer capacity [20]. Niranjan V found that TLC, FVC, FEV1, and VC values were significantly lower in patients with type 1 diabetes than in healthy subjects [21]. The results pertaining to the correlations between $\mathrm{HbA1c}$ and pulmonary function that were noted in previous studies were inconsistent. Two studies noted weak associations between HbA1c and spirometric parameters and strong correlations between diabetes duration and pulmonary function [22,23]. Another cross-sectional population study noted that plasma glucose levels were negatively correlated with FVC and/or FEV [24].

The precise mechanisms underlying DKD development are unknown; however, several theories exist regarding the specific processes that affect haemodynamics in DKD. Haemodynamic changes in diabetic kidneys have been the focus of considerable research. Renin-angiotensin system (RAS) activation reportedly 
induces intrarenal haemodynamic abnormalities in diabetes [25]. Taniwaki $\mathrm{H}$ and colleagues demonstrated that the intrarenal RAS may be activated in diabetes and subsequently facilitate increases in the RI and that RAS activation may be impacted by poor glycaemic control. In addition, these authors showed that blocking RAS activation with captopril may reduce intrarenal vascular resistance in diabetes [26].

Elevated RIs have been reported to be associated with vascularinterstitial diseases, including DKD (but not glomerulopathies). Increased RIs may be reflective of decreased tissue and vascular compliance, as well as increased vascular resistance [9]. However, the early stages of DN are associated with an increased GFR and variable increases in renal plasma flow and the filtration fraction in both clinical and experimental settings. Diabetic hyper perfusion and hyperfiltration at the nephron level are characterized by disproportionate decreases in afferent arteriolar resistance. These changes may also be reflected by increased RIs. RIs are measured by duplex Doppler sonography [27]. Biopsy studies involving children have shown that basement membrane thickening and mesangial expansion in the kidney develop prior to the onset of microalbuminuria [28]. Doppler sonography apparently does not replace renal biopsy but is a readily applicable and noninvasive tool for investigating renal haemodynamics and a credible means of exploring renal structures for the purpose of collecting both morphologic and physiologic data for the study of renal blood flow in children [29]. To our knowledge, our study is the first to use the RI to predict changes in renal function in the preclinical stage of DKD, results similar to those of the study by Pelliccia P, which involved children [29].

However, there is no general agreement with respect to the significance and predictive value of the renal RI in patients with DKD. Researchers have performed several studies regarding the application of Doppler sonography for the evaluation of intrarenal haemodynamic abnormalities in adults with DKD [9,30]; however, studies regarding the preclinical stage of DKD (in which renal function is normal) in adults are still lacking. In our study, we aimed to explore whether Doppler sonography could be used to detect alterations in the renal RI in adults with diabetes who had normal renal function.

Table 1: The change of RI in the interlobular renal arteries and the pulmonary function from baseline to year 15.

\begin{tabular}{|c|c|c|c|c|c|}
\hline \multirow{2}{*}{\multicolumn{2}{|c|}{$\begin{array}{l}\text { Content } \\
\mathrm{A}(\leq \mathbf{0 . 7})\end{array}$}} & \multicolumn{2}{|c|}{ Group (mean RI) } & \multirow{2}{*}{$t / \chi^{2}$ value } & \multirow{2}{*}{$P$ value } \\
\hline & & $\mathrm{B}(>0.7)$ & & & \\
\hline \multicolumn{2}{|c|}{ NO. $(n)$} & 25 & 35 & - & - \\
\hline \multirow{2}{*}{ Sex, $n(\%)$} & Male & $13(52.00)$ & 19 (54.29) & \multirow{2}{*}{0.031} & \multirow{2}{*}{0.861} \\
\hline & Female & $12(48.00)$ & $16(45.71)$ & & \\
\hline \multicolumn{2}{|c|}{ Age (years) in 2000} & $52.44 \pm 5.77$ & $52.49 \pm 5.64$ & 0.031 & 0.976 \\
\hline \multicolumn{2}{|c|}{ BMI $\left(\mathrm{kg} / \mathrm{m}^{2}\right)$ in 2000} & $27.44 \pm 1.36$ & $27.31 \pm 1.64$ & 0.314 & 0.755 \\
\hline \multicolumn{2}{|c|}{ Diabetes duration (years) in 2000} & $7.65 \pm 2.21$ & $7.27 \pm 1.76$ & 0.741 & 0.462 \\
\hline DKD, $n(\%)$ & DKD & $6(24.00)$ & $23(65.71)$ & \multirow{2}{*}{10.162} & \multirow{2}{*}{$0.001^{*}$} \\
\hline in the 2015 & NDKD & $19(76.00)$ & $12(34.29)$ & & \\
\hline \multirow{3}{*}{$\begin{array}{c}\text { Mean RI in interlobular renal } \\
\text { arteries }\end{array}$} & baseline & $0.67 \pm 0.02$ & $0.75 \pm 0.02$ & 15.29 & $0.000^{*}$ \\
\hline & year 15 & $0.71 \pm 0.03$ & $0.80 \pm 0.03$ & 12.202 & $0.000^{*}$ \\
\hline & change & $0.05 \pm 0.01$ & $0.05 \pm 0.02$ & 0.451 & 0.653 \\
\hline \multirow{3}{*}{ AER } & baseline & $16.88 \pm 1.48$ & $19.97 \pm 1.20$ & 8.919 & $0.000^{*}$ \\
\hline & year 15 & $24.84 \pm 7.06$ & $35.00 \pm 6.85$ & 5.591 & $0.000^{*}$ \\
\hline & change & $7.96 \pm 6.40$ & $15.03 \pm 6.60$ & 4.142 & $0.000^{*}$ \\
\hline \multirow{3}{*}{ UACR } & baseline & $16.04 \pm 1.54$ & $18.74 \pm 1.24$ & 7.507 & $0.000^{*}$ \\
\hline & year 15 & $27.16 \pm 5.68$ & $34.46 \pm 6.02$ & 4.741 & $0.000^{*}$ \\
\hline & change & $11.12 \pm 4.96$ & $15.71 \pm 5.34$ & 3.383 & $0.000^{*}$ \\
\hline \multirow{3}{*}{$\mathrm{Cr}$} & baseline & $74.16 \pm 5.47$ & $75.34 \pm 4.19$ & 0.949 & 0.347 \\
\hline & year 15 & $87.72 \pm 7.46$ & $92.26 \pm 5.20$ & 2.777 & $0.007^{*}$ \\
\hline & change & $13.56 \pm 5.82$ & $16.91 \pm 4.52$ & 2.511 & $0.015^{*}$ \\
\hline \multirow{3}{*}{ BUN } & baseline & $5.49 \pm 0.59$ & $5.90 \pm 0.35$ & 3.313 & $0.002^{*}$ \\
\hline & year 15 & $6.43 \pm 0.49$ & $6.78 \pm 0.44$ & 2.911 & $0.005^{*}$ \\
\hline & change & $0.94 \pm 5.47$ & $0.89 \pm 0.44$ & 0.42 & 0.676 \\
\hline \multirow{3}{*}{ VC (\% of predicted) } & baseline & $80.24 \pm 1.67$ & $76.60 \pm 2.34$ & 6.656 & $0.000^{*}$ \\
\hline & year 15 & $76.12 \pm 2.11$ & $71.34 \pm 2.21$ & 8.416 & $0.000^{*}$ \\
\hline & change & $-4.12 \pm 1.36$ & $-5.26 \pm 1.82$ & 2.637 & $0.011^{*}$ \\
\hline
\end{tabular}




\begin{tabular}{|c|c|c|c|c|c|}
\hline \multirow{3}{*}{ FVC (\% of predicted) } & baseline & $72.64 \pm 2.34$ & $69.46 \pm 3.27$ & 4.163 & $0.000^{*}$ \\
\hline & year 15 & $68.76 \pm 2.68$ & $64.37 \pm 3.34$ & 5.429 & $0.000^{*}$ \\
\hline & change & $-3.88 \pm 2.30$ & $-5.09 \pm 2.32$ & 1.994 & 0.051 \\
\hline \multirow{3}{*}{ FEV1 (\% of predicted) } & baseline & $77.64 \pm 3.01$ & $73.26 \pm 1.29$ & 7.694 & $0.000^{*}$ \\
\hline & year 15 & $73.20 \pm 4.25$ & $68.20 \pm 2.15$ & 5.979 & $0.000^{*}$ \\
\hline & change & $-4.44 \pm 1.85$ & $-5.06 \pm 1.47$ & 1.437 & 0.156 \\
\hline \multirow{3}{*}{ PEF (\% of predicted) } & baseline & $51.12 \pm 3.13$ & $48.57 \pm 1.52$ & 4.162 & $0.000^{*}$ \\
\hline & year 15 & $47.04 \pm 3.71$ & $43.66 \pm 1.75$ & 4.718 & $0.000^{*}$ \\
\hline & change & $-4.08 \pm 2.04$ & $-4.91 \pm 1.52$ & 1.816 & 0.075 \\
\hline \multirow{3}{*}{ MVV (\% of predicted) } & baseline & $88.04 \pm 2.64$ & $84.89 \pm 1.91$ & 5.382 & $0.000^{*}$ \\
\hline & year 15 & $81.96 \pm 2.72$ & $77.54 \pm 2.82$ & 6.072 & $0.000^{*}$ \\
\hline & change & $-6.08 \pm 1.55$ & $-7.34 \pm 2.72$ & 2.087 & $0.041^{*}$ \\
\hline \multirow{3}{*}{ TLC (\% of predicted) } & baseline & $92.64 \pm 3.84$ & $88.54 \pm 2.05$ & 5.348 & $0.000^{*}$ \\
\hline & year 15 & $88.84 \pm 4.57$ & $83.66 \pm 2.54$ & 5.613 & $0.000^{*}$ \\
\hline & change & $-3.80 \pm 1.19$ & $-4.89 \pm 1.78$ & 2.654 & $0.010^{*}$ \\
\hline \multirow{3}{*}{ FEV1/FVC (\% of predicted) } & baseline & $74.48 \pm 1.92$ & $71.17 \pm 1.36$ & 7.825 & $0.000^{*}$ \\
\hline & year 15 & $71.00 \pm 2.25$ & $67.63 \pm 1.57$ & 6.829 & $0.000^{*}$ \\
\hline & change & $-3.48 \pm 1.33$ & $-3.54 \pm 1.54$ & 0.165 & 0.87 \\
\hline \multirow{3}{*}{ DLCO (\% of predicted) } & baseline & $84.84 \pm 2.41$ & $82.03 \pm 2.22$ & 4.672 & $0.000^{*}$ \\
\hline & year 15 & $81.60 \pm 3.18$ & $77.03 \pm 2.35$ & 6.419 & $0.000^{*}$ \\
\hline & change & $-3.24 \pm 1.76$ & $-5.00 \pm 1.78$ & 3.788 & $0.000^{*}$ \\
\hline \multirow{3}{*}{ DLCO/VA (\% of predicted) } & baseline & $88.08 \pm 3.58$ & $83.06 \pm 1.68$ & 7.27 & $0.000^{*}$ \\
\hline & year 15 & $85.60 \pm 4.45$ & $78.71 \pm 1.84$ & 8.237 & $0.000^{*}$ \\
\hline & change & $-2.48 \pm 1.83$ & $-4.34 \pm 1.51$ & 4.308 & $0.000^{*}$ \\
\hline
\end{tabular}

Note: ${ }^{*} P<0.05$, the difference between the two groups had statistical significance.

In our study, the results of the longitudinal follow-up study, in which pulmonary function was assessed in patients organized into separate groups according to their RIs, indicated that subjects with RIs $\leq 0.7$ displayed significantly better pulmonary function in 2000 than subjects with RIs $>0.7$. Moreover, pulmonary function showed improvement and decreased to a smaller extent in the former group compared to the latter group from 2000 to 2015. Additionally, the indicated renal functional parameters (BUN and $\mathrm{Cr}$ ) and the AER and UACR showed improvement and increased to a smaller extent in the former group compared to the latter group from 2000 to 2015. The incidence of DKD in the group with RIs $\leq 0.7$ (6/25, 24.00\%) was significantly lower than that in the group with RIs $>0.7(23 / 45$, 65.71\%) $(\mathrm{P}<0.05)$ (Table 1).

In our study, we demonstrated that pulmonary functional parameters were negatively correlated with the bilateral kidney RI; however, our study had several limitations that should be addressed in future studies. First, we failed to observe the changes in alveolar tissue morphology associated with diabetes and did not identify the specific protein responsible for inducing the changes. Because not all the patients underwent a lung biopsy, we had to adopt an animal model to study alveolar tissue samples. Second, we did not assess inflammatory factors or ROS, both of which are associated with lung dysfunction in T2DM [16-18]. It is worth evaluating whether they can be used to predict pulmonary function and renal function during the preclinical stage of DKD. Therefore, we recommend that clinicians monitor patients with T2DM for signs of lung damage in addition to monitoring them for signs of DKD and DR.

\section{Conclusion}

In conclusion, According to the above results, we surmised that the kidney RI may be used not only to evaluate the haemodynamic changes characteristic of DKD and the progression of the disease but also to predict pulmonary and renal function in adults and children during the preclinical stage of DKD. Thus, in addition to the HbA1c and diabetes duration, the renal RI, can also be used to predict the likelihood of DKD during the preclinical stage of the disease in question.

\section{Acknowledgement}

The authors would like to thank all of the patients and their families, the team of investigators, research nurses, and operations staff involved in this study. Editorial support (in the form of writing assistance, including development of the initial draft based on author input, assembling tables and figures, collating authors comments, grammatical editing and referencing) was provided by He Tai. The translator of English was provided by American journal experts. 


\section{Availability of Data and Materials}

The data from the original study are available from Shenyang the Fourth Hospital of People via its ClinicalTrials.gov (NCT02798198). The datasets analyzed during the current study are available from all the authors on reasonable request.

\section{Ethics Approval and Consent to Participate}

The study complied with the Declaration of Helsinki and the International Conference on Harmonisation and Good Clinical Practice guidelines. The protocol was approved by the regulatory authority for each country (where applicable) and an independent ethics committee at each center. and the clinical research protocol was approved by the medical ethics committee (number ICE20160806) of our hospital. Each subject who participated in the study or one of their family members provided written informed consent authorizing their enrolment herein.

\section{Conflict of Interest}

Author declare no conflict of interest.

\section{References}

1. Ronald CW Ma, Juliana CN Chan (2013) Type 2 diabetes in East Asians: similarities and differences with populations in Europe and the United States. Ann N Y Acad Sci 1281: 64-91.

2. Lin CH, Chang DM, Wu DJ, Peng HY, Chuang LM (2015) Assessment of Blood Glucose Regulation and Safety of Resistant Starch Formula-Based Diet in Healthy Normal and Subjects with Type 2 Diabetes. Medicine (Baltimore) 94(33): e1332.

3. Murea M, Ma L, Freedman BI (2012) Genetic and environmental factors associated with type 2 diabetes and diabetes vascular complications. Rev Diabet Stud 9(1): 6-22.

4. Yamagishi S, Fukami K, Matsui T (2015) Crosstalk between advanced glycation end products (AGEs)-receptor RAGE axis and dipeptidyl peptidase-4-incretin system in diabetes vascular complications. Cardiovasc Diabetol 14: 2.

5. Kodolova IM, Lysenko IV, Saltykov BB (1982) Change in the lung in diabetes mellitus. Arkh Pathol 44(7): 35-40.

6. Kwon CH, Rhee EJ, Song Jk, Kim JT, Kwag HJ, et al. (2012) Reduced lung function is independently associated with increased risk of type 2 diabetes in Korean men. Cardiovasc Diabetol 11: 38.

7. Goebel W, Lieb WE, Ho A, Sergott RC, Farhoumand R, et al. (1995) Color Doppler imaging: a new technique to assess orbital blood flow in patients with diabetes retinopathy. Invest Ophthalmol Vis Sci 36(5): 864-870.

8. Dimitrova G, Kato S, Fukushima H, Yamashita H (2009) Circulatory parameters in the retrobulbar central retinal artery and vein of patients with diabetes and medically treated systemic hypertension. Graefes Arch Clin Exp Ophthalmol 247(1): 53-58.

9. Tublin ME, Bude RO, Platt JF (2003) The resistive index in renal Doppler sonography: where do we stand? AJR Am J Roentgenol 180(4): 885-892.

10. American Diabetes Association (2011) Standards of medical care in diabetes 2007. Diabetes Care 30 (Suppl. 1): S11-S61.

11. Stevens LA, Coresh J, Schmid CH, Feldman HI, Froissart M, et al. Estimating GFR using serum cystatin $\mathrm{C}$ alone and in combination with serum creatinine: a pooled analysis of 3,418 individuals with CKD. Am J Kidney Dis, 2008; 51(3): 395-406.
12. Frauchiger B, Nussbaumer P, Hugentobler M, Staub D ( 2000) Duplex sonographic registration of age and diabetes-related loss of renal vasodilatatory response to nitroglycerine. Nephrol Dial Transplant 15(6): 827-832.

13. Veglio F, Francisco M, Melchio R, Provera E, Rabbia F, et al. ( 1995) Assessment of renal resistance index after captopril test by Doppler in essential and renovascular hypertension. Kidney Int 48(5): 1611-1616.

14. Cavan DA, Parkes A, O’Donnell MJ, Freeman W, Cayton RM (1991) Lung function and diabetes. Respir Med 85(3): 257-258.

15. Hsia CC, Raskin P (2007) Lung function changes related to diabetes mellitus. Diabetes Technol Ther. 9 (Suppl 1): S73-S82.

16. Ford ES (1999) Body mass index, diabetes, and C-reactive protein among U.S. adults. Diabetes Care, 22(12): 1971-1977.

17. Rodriguez M, Guerrero R (1999) Increased levels of C-reactive protein in non-controlled type 2 diabetes subjects. J Diabetes Complications 13(4): 211-215.

18. Tan K, Chow W, Tam S, Ai VH, Lam CH, et al. (2002) Atorvastatin lowers C-reactive protein and improves endothelium-dependent vasodilation in type 2 diabetes mellitus. J Clin Endocrinol Metab 87(2): 563 -568.

19. Barnes PJ (2002) The role of inflammation and anti-inflammatory medication in asthma. Respir Med, 96 (Suppl. A): S9-S15.

20. Sandler M, Bunn A, Stewart R , (1987) Cross-section study of pulmonary function in patients with insulin-dependent diabetes mellitus. Am Rev Respir Dis 135(1): 223-228.

21. Niranjan V, McBrayer DG, Ramirez LC, Raskin P, Hsia CC (1997) Glycemic control and cardiopulmonary function in patients with insulindependent diabetes mellitus. Am J 103(6): 504-513.

22. Davis TM, Knuiman M, Kendall P, Vu H, Davis WA (2000) Reduced pulmonary function and its associations in type 2 diabetes: the Fremantle Diabetes Study. Diabetes Res Clin Pract 50(2): 153-159.

23. Barrett-Connor E, Frette C (1996) NIDDM, impaired glucose tolerance, and pulmonary function in older adults. The Rancho Bernardo Study. Diabetes Care 19(12): 1441-1444.

24. Lange P, Groth S, Kastrup J, Mortensen J, Appleyard M, et al. (1989) Diabetes mellitus, plasma glucose and lung function in a cross-sectional population study. Eur Respir J, 2(1): 14-19.

25. Miller JA (1999) Impact of hyperglycemia on the renin angiotensin system in early human type 1 diabetes mellitus. J Am Soc Nephrol, 10(8): 1778-1785.

26. Taniwaki Hi, Ishimura E, Kawagishi T, Matsumoto N, Hosoi M et al. Intrarenal hemodynamic changes after captopril test in patients with type 2 diabetes: a duplex Doppler sonography study. Diabetes Care, 2003; 26(1): 132-137.

27. Matsumoto N, Ishimura E, Taniwaki H, Emoto M, Shoji T, et al. (2000) Diabetes mellitus worsens intrarenal hemodynamic abnormalities in non-dialyzed patients with chronic renal failure. Nephron 86(1): 44-51.

28. Pelliccia P, Savino A, Cecamore C, Primavera A, Schiavone C, et al. (2008) Early Changes in Renal Hemodynamics in Children with Diabetes: Doppler Sonographic Findings. J Clin Ultrasound, 36(6): 335-340.

29. Ellis EN, Warady BA, Wood EG, Hassanein R, Richardson WP, et al.(1997) Renal structural-functional relationships in early diabetes mellitus. Pediatr Nephrol 11(5): 584-591.

30. Ohta Y, Fujii K, Arima H, Matsumura K, Tsuchihashi T, et al. (2005) Increased renal resistive index in atherosclerosis and diabetes nephropathy assessed by Doppler sonography. J Hypertens 23(10): 1905-1910. 